\title{
Décadrages Décadrages
}

cinéma, à travers champs Cinéma, à travers champs

13 | 2008

Anna Sanders Films, cinéma et art contemporain

\section{Un autre homme qui tient toutes ses promesses}

\section{Xavier Reymond}

\section{OpenEdition}

Journals

Édition électronique

URL : http://journals.openedition.org/decadrages/525

DOI : $10.4000 /$ decadrages. 525

ISSN : 2297-5977

\section{Éditeur}

Association Décadrages

\section{Édition imprimée}

Date de publication : 10 octobre 2008

Pagination : 95-98

ISBN : 978-2-9700582-8-1

ISSN : 2235-7823

Référence électronique

Xavier Reymond, "Un autre homme qui tient toutes ses promesses », Décadrages [En ligne], 13 | 2008, mis en ligne le 10 octobre 2009, consulté le 22 septembre 2020. URL : http://journals.openedition.org/ decadrages/525 ; DOI : https://doi.org/10.4000/decadrages.525

Ce document a été généré automatiquement le 22 septembre 2020

(B) Décadrages 


\title{
Un autre homme qui tient toutes ses promesses
}

\author{
Xavier Reymond
}

1 Un auditorium FEVI plein à craquer a accueilli le 9 août 2008 à Locarno le baptême public du dernier-né de Lionel Baier, le «jeune cinéaste suisse le plus prometteur du moment $\rrbracket^{1}$, non sans une certaine appréhension toutefois. En effet, s'ils avaient su faire parler d'eux en divisant un public partagé entre l'attachement pour un dynamisme revigorant couplé à un regard original, et l'agacement face à un nombrilisme à la limite de l'arrogance, ses précédents essais de fiction, Garçon stupide (2004) et Comme des voleurs (à l'est) (2006), pâtissaient de maladresses, tant sur le plan de la construction de l'intrigue que du traitement de l'image. Les festivaliers attendaient donc avec impatience Un autre homme, susceptible de confirmer pour de bon cette fois le talent indéniable de son réalisateur, et de rendre justice à sa formidable énergie. Quatrevingt-onze minutes de projection plus tard, après avoir ri tantôt jaune, tantôt à gorge déployée, le public conquis applaudissait à tout rompre le réalisateur et son équipe, augurant du meilleur pour l'avenir du film.

2 Bien qu'absent du palmarès de la compétition officielle, dont, il est vrai, les œuvres primées explorent des thématiques assez différentes, Un autre homme devrait tout de même marquer l'histoire du cinéma romand d'une (petite) pierre blanche. En effet, s'il n'annonce peut-être pas encore une nouvelle étape du mouvement de fond lancé dans ce microcosme par Vincent Pluss avec On dirait le sud (2002) $)^{2}$, ce film, comme nous allons tenter ici de le montrer en nous arrêtant sur certains de ses aspects les plus remarquables, témoigne aussi bien de la maturité artistique atteinte par Lionel Baier, que de son indépendance jalousement défendue. Le septième art romand peut donc célébrer le passage de ce cinéaste du stade d'espoir prometteur au rang d'auteur sur lequel il faut compter.

3 Dressant le portrait et suivant l'évolution d'un jeune homme, François, dont l'arrogance s'associe au manque de confiance en soi pour en faire un personnage tout à la fois détestable et attachant, l'intrigue du film surprend en premier lieu par un des sujets qu'elle se propose de traiter (aux côtés de l'arrivisme social, de la passivité 
masculine et de l'élitisme urbain notamment) : la critique de cinéma. Si ce n'est pas la première fois qu'un critique occupe le premier rôle d'un film³ , le métier lui-même n'a sans doute jamais connu pareille description en profondeur, qui nous emmène des projections de presse aux émissions de radio, en passant par les conversations teintées de suffisance et de futilité qui caractérisent les rapports entre les personnages de ce milieu. Particulièrement acide, et du coup souvent très drôle, la satire permet de soulever plusieurs questions importantes concernant la critique de cinéma, et même d'art en général, interrogeant crûment sa nécessité, sa pertinence, ou encore sa sincérité. Si l'originalité d'un tel sujet peut sembler attirante à première vue, la moindre maladresse aurait eu vite fait de transformer ce long métrage en un objet prétentieux et fort désagréable, constituant un véritable cadeau empoisonné pour son réalisateur. Or, miracle, tout fonctionne ici à merveille, et si Lionel Baier ne se montre certes pas tendre envers les critiques en leur tendant un miroir fort peu charmeur, ses nombreuses piques atteignent leur cible avec une classe toute particulière.

4 Le noir et blanc choisi pour habiller cette comédie acide mérite aussi d'être applaudi, en premier lieu peut-être pour ses grandes qualités esthétiques. Couplé à un éclairage très soigné, parfois un peu crû mais jamais obscène, qu'il s'agisse de faire apparaître à l'écran des corps dénudés ou des paysages glacés, il procure un plaisir visuel auquel les précédentes œuvres de Lionel Baier ne nous avaient guère habitués, prenant prétexte d'un style docu-fiction assumé pour négliger quelque peu la photographie. Ce souci du beau ne se veut cependant pas gratuit, puisqu'il est soumis ici de manière cohérente aux exigences du récit. En effet, les nombreuses oppositions autour desquelles l'intrigue se noue trouvent dans le noir et blanc de l'image leur traduction naturelle. Qu'il s'agisse de contrastes régionaux, sociaux ou humains, leur matérialisation sur un écran monochrome ne les rend que plus frappants encore.

5 Cet écrin photographique permet à Lionel Baier de nous offrir alors l'une des plus belles et inventives mises en images de Lausanne qui soient, démontrant à nouveau chez l'auteur une excellente maîtrise du langage filmique. Des travellings sur les façades de l'avenue des Terreaux, déjà essayés avec succès dans Garçon stupide (2004), aux panoramiques du sommet du beffroi de la cathédrale, en passant par une visite de la tour Edipresse ou du pont Bessières, tous les plans contribuent à poser un décor fait de mouvement, de verticalité, et d'équilibre précaire pour les personnages qui y évoluent, constituant ainsi une métaphore visuelle convaincante de l'histoire d'ascension sociale qui s'y trame. Si le procédé paraîtrait se situer à la limite du cliché dans une ville telle que New York, il frappe ici par son originalité, tant l'image habituelle de la capitale vaudoise se révèle d'ordinaire stéréotypée du côté du provincialisme et du pittoresque. Si les spectateurs lausannois seront sans doute surpris par ce regard différent, les personnes étrangères à la cité penseront peut-être que le récit s'adapte parfaitement à la ville de tournage choisie, alors même que c'est cette ville qui, à travers la caméra de Lionel Baier, ne cesse de s'adapter à l'intrigue.

6 Enfin, la manière dont la sexualité est mise en scène mérite également quelques éloges. Si le sexe se taille une place importante à l'écran, il ne s'agit absolument pas ici de pornographie, ni même d'érotisme. D'une part, la nudité impudique de François filmé dans son domicile participe à la fois de la création d'une certaine intimité entre le spectateur et le personnage, et d'une volonté manifeste du réalisateur de ne pas céder aux tabous usuels concernant les parties du corps qui doivent ou ne doivent pas être montrées au cinéma, selon que l'on filme un homme ou une femme. D'autre part, la 
relation à tendance sadomasochiste que vit François avec une critique contribue grandement à la construction d'un portrait d'homme passif, sorte d'équivalent masculin de la femme-objet, dont la psychologie particulière constitue l'un des intérêts principaux du film. Loin de s'immiscer dans les images pour satisfaire un quelconque plaisir voyeuriste, les scènes de sexe correspondent donc à des fonctions bien précises du récit, que Lionel Baier entend développer sans pudibonderie ni gratuité, en suivant ses propres codes, qui ne s'apparentent pas nécessairement aux normes sociales habituelles. Une telle attitude, assez courageuse puisqu'elle pourrait mettre en cause le potentiel commercial du film, résonne à nouveau comme une promesse d'originalité et d'indépendance d'esprit.

7 A Locarno, la critique francophone a réservé une véritable fête à Lionel Baier, saluant tour à tour les qualités narratives et esthétiques d'Un autre homme, ainsi que son audace et son originalité. «Meilleur film $»^{4}$ de son auteur pour Le Temps, il est l'œuvre d'un de ces réalisateurs qui «choisissent de se moquer ouvertement d'eux-mêmes et y parviennent brillamment $»^{5}$ pour Le Monde, alors que L'Hebdo préfère souligner son caractère divertissant en le taxant de "drôle, qui ne se réclame pas du comique lourdingue [...], mais plutôt de l'esprit de Flaubert $»^{6}$. Enfin, au-delà du film, Patrick Ferla à la Radio Suisse Romande met peut-être le doigt sur le trait de caractère le plus remarquable de Lionel Baier : son envie intense de cinéma, qui implique que «là où d'autres renoncent, lui entreprend, là où d'autres se lassent, lui opte pour le désir » ${ }^{7}$. En d'autres mots, au lieu de laisser se consumer cette envie de cinéma à petit feu en attendant pour mettre un projet sur pied un soutien des pouvoirs publics forcément hypothétique, Lionel Baier choisit délibérément la prise de risque et tourne son film au plus vite, seul, sans même attendre un quelconque signal de l'office fédéral de la culture. Obtenir un résultat aussi proche de l'excellence dans de telles conditions constitue assurément un véritable tour de force, auquel il faut espérer que le public, suisse et international, saura réserver l'accueil qu'il mérite.

Un autre homme (CH, 2008, 91').

Réalisation, scénario et image : Lionel Baier.

Montage : Pauline Gaillard. Son : Thibault de Chateauvieux, Kaveh Bakhtiari, Joëlle

Bacchetta, Laurent Gabiot.

Interprètes : Robin Harsch, Natacha Koutchoumov, Bulle Ogier,...

\section{NOTES}

1. Olivier Séguret, Libération, 14 août 2008.

2. Maria Tortajada, «A nouveau du nouveau dans le cinéma suisse. L'affaire Vincent Pluss et le cinéma romand ", Décadrages 1-2. Cinéma à travers champs, 2003.

3. Rien sur Robert (Pascal Bonitzer, 1999), par exemple, narre les mésaventures consécutives à un article malheureux d'un critique de cinéma (interprété par Fabrice Luchini), dont la personnalité ne manque d'ailleurs pas de points communs avec celle de François. 
4. Thierry Jobin, Le Temps, 12 août 2008.

5. Jacques Mandelbaum, Le Monde, 19 août 2008.

6. Antoine Duplan, L'Hebdo, 9 août 2008. http://dua.typepad.com/cinema/2008/08/locarnojour-4-lheure-de-baier.html, consulté le 18 septembre 2008.

7. Patrick Ferla, Radio Suisse Romande, 9 août 2008. http://zoom.rsr.ch/?p=989, consulté le 18 septembre 2008. 